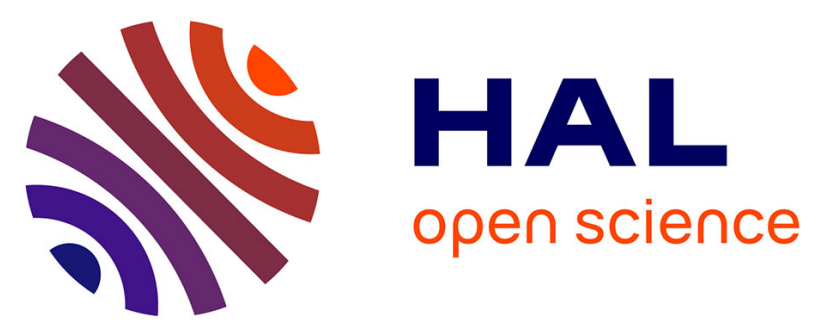

\title{
Comparison of optical sensors discrimination ability using spectral libraries
}

Germain Forestier, Jordi Inglada, Cédric Wemmert, Pierre Gancarski

\section{To cite this version:}

Germain Forestier, Jordi Inglada, Cédric Wemmert, Pierre Gancarski. Comparison of optical sensors discrimination ability using spectral libraries. International Journal of Remote Sensing, 2013, 34 (7), pp.2327 - 2349. 10.1080/01431161.2012.744488 . hal-01875856

\section{HAL Id: hal-01875856 https://hal.science/hal-01875856}

Submitted on 17 Sep 2018

HAL is a multi-disciplinary open access archive for the deposit and dissemination of scientific research documents, whether they are published or not. The documents may come from teaching and research institutions in France or abroad, or from public or private research centers.
L'archive ouverte pluridisciplinaire HAL, est destinée au dépôt et à la diffusion de documents scientifiques de niveau recherche, publiés ou non, émanant des établissements d'enseignement et de recherche français ou étrangers, des laboratoires publics ou privés. 
This is the author's version of an article published in the International Journal of Remote Sensing. The final authenticated version is available online at: https://doi.org/10. $1080 / 01431161.2012 .744488$.

\title{
Comparison of optical sensors discrimination ability using spectral libraries
}

\author{
Germain Forestier $^{1 *}$, Jordi Inglada ${ }^{2}$, Cédric Wemmert ${ }^{3}$ and Pierre Gançarski ${ }^{3}$ \\ ${ }^{1}$ University of Haute Alsace, MIPS - EA2332, Mulhouse, France \\ ${ }^{2}$ CESBIO (CNES/CNRS/UPS/IRD), Toulouse, France \\ ${ }^{3}$ University of Strasbourg, LSIIT-CNRS UMR7005, Strasbourg, France
}

(Received 00 Month 20XX; accepted 00 Month 20XX)

\begin{abstract}
In remote sensing, the ability to discriminate different land covers or material types is directly linked with the spectral resolution and sampling provided by the optical sensor. Previous studies showed that the spectral resolution is a critical issue, especially in complex environment. In spite of the increasing availability of hyperspectral data, multispectral optical sensors onboard various satellites are acquiring everyday a massive amount of data with a relatively poor spectral resolution (i.e. usually about 4 to 7 spectral bands). These remotely sensed data are intensively used for Earth observation regardless of their limited spectral resolution. In this paper, we studied seven of these optical sensors: Pleiades, QuickBird, SPOT5, Ikonos, Landsat TM, Formosat and Meris. This study focuses on the ability of each sensor to discriminate different materials according to its spectral resolution. We used four different spectral libraries which contains around 2500 spectra of materials and land covers with a fine spectral resolution. These spectra were convolved with the Relative Spectral Responses (RSR) of each sensor to create spectra at the sensors' resolutions. Then, these reduced spectra were compared using separability indexes (Divergence, Transformed divergence, Bhattacharyya, Jeffreys-Matusita) and machine learning tools. In the experiments, we highlighted that the spectral bands configuration could lead to important differences in classification accuracy according to the context of application (e.g. urban area).
\end{abstract}

\section{Introduction}

The spectral resolution of a sensor can be characterized by the number of spectral bands, their associated bandwidths and their locations along the spectrum (Herold, Gardner, and Roberts 2003). Spectral resolution is also described by Lillesand and Kiefer (1994) as "the ability to discriminate fine spectral differences". Several previous studies (Herold, Gardner, and Roberts 2003; Meyer and Chander 2007; Heidena et al. 2007) showed that the spectral resolution is a critical issue, especially to discriminate different land covers in complex environment. Most of multispectral systems have 4 to 7 spectral bands within the visible to middle infrared region of the electromagnetic spectrum. There exist however some systems that use one or more thermal infrared bands. One of the main benefits to use only multispectral versus hyperspectral acquisition, is the larger spatial coverage, which allows a faster and wider mapping of large areas. Indeed, satellite remote sensing systems provide both, a synoptic view space and economies of scale (Govender, Chetty, and

*Corresponding author. Email: germain.forestier@uha.fr 
Bulcock 2007). Several satellites are now available on the market, each one offering specificities in terms of spectral acquistion according to the parameters its sensors. Consequently, it is sometime difficult to choose a well suited sensor for a specific remote sensing application. For example, multi-source approaches (Wemmert et al. 2009; Forestier, Wemmert, and Gancarski 2008) try to combine information provided by several sensors, however it is often difficult to chose the sensors that can benefit from each others. To address this issue, simulation approaches can be used in order to perform a theoretical comparison of the ability of several sensors to discriminate fine spectral differences. In this paper, we used several spectral libraries which contain spectra of materials and land covers with a fine spectral resolution. These spectra were extracted from the libraries and convolved with the Relative Spectral Responses (RSR) of several sensors to create coarser spectra at the sensors' spectral resolutions. This work focuses on the ability of each sensor to discriminate different materials, according to its spectral resolution. As the simulated spectra for each sensor are created from exactly the same precise spectra extracted from the libraries, the only variation is the RSR of the sensors. Thus, we only focus on comparing the spectral resolution of the sensors as we do not take into account the spatial resolution of the sensor, or other external factors (e.g. atmosphere, acquisition noise, etc.). While this choice limits the scope of this study, we believe that the insights provided by spectral comparison already bring an interessing knowledge about the discrimination ability of the studied sensors. Furthermore, we show that simulation can also be used fro other applications, from evaluating the interest of a spectral index (e.g. NDVI) to the design of new sensors.

\section{Sensor simulation}

\subsection{Previous work}

Sensor simulation, also called band simulation or band synthesis, consists in generating simulated multispectral spectra from data acquired by existing sensors, but with higher spectral resolution. The simulation consists in combining hyperspectral narrow bands into broader multispectral bands. This kind of approach has already been used, especially for sensor calibration and sensor simulation. The spectra simulation step uses the Relative Spectral Response (RSR) functions of the multispectral sensor, which describe the spectral response of each simulated sensor's band. For example, Green and Shimada (1997) used band synthesis for cross-calibration of a satellite multispectral instrument using AVIRIS data. The simulated data were used to determine the on-orbit radiometric calibration coefficients required for calibration of the spectral bands of the satellite.

A similar approach was used by Chander, Meyer, and Helder (2004) for the cross-calibration of the Advanced Land Imager (ALI) from Landsat ETM+ wellcalibrated data.

Salvatore et al. (1999) used band simulation to simulate the response of a new sensor from AVIRIS data. This simulation allowed the investigators to evaluate in advance the potentialities of the new multispectral sensor. This kind of simulation provides an opportunity to try variations in the original spectral response, and to adjust the RSR to achieve better results for the multispectral sensor objectives.

In another study, Jarecke et al. (2001) used Hyperion hyperspectral data to simulate Landsat ETM+ data and concluded that the difference between the simulated data and real data actually acquired by Landsat ETM+ was around $10 \%$. Meyer and Chander (2007) used AVIRIS data to simulate MODIS and Landsat ETM+ 
data in order to illustrate how the differences in the RSR affect the observation of some typical surface features. The authors noticed some strong variations according to the different RSR. They concluded that more investigations were needed on how RSR affects the observation of different surfaces.

Concerning spectral indexes, Franke, Heinzel, and Menz (2006) used band simulation to compare the differences of the Normalized Difference Vegetation Index (NDVI) according to the RSR of Landsat TM, Quickbird and SPOT5-HRV. The assessment of NDVI differences showed substantial variations between sensor systems. An intercalibration approach using a polynomial order is suggested, to adjust NDVI differences caused by varying RSR functions. Polynomial corrections are also suggested for normalization. Trishchenko (2009) also studied the effects of RSR on surface reflectance and NDVI measured with moderate resolution satellite sensors. The results show more consistency between sensors with typical correction being under 5\%. Another study of Teillet, Staenz, and William (1997) demonstrates the impact of changes in RSR on NDVI derived from AVIRIS data. The results indicate that the NDVI is significantly affected by differences in spectral bandwidth, especially for the red band, and that changes in spatial resolution lead to less persuasive but more land cover specific effects on NDVI. More recently, Pandya et al. (2007) published a report on experiments to estimate and compare effective spectral characteristics of various spectral channels and to quantify the influence of varying sensor spectral response on reflectance and NDVI. They observed a significant deviation in the central wavelength and the spectral width for the corresponding channel of IRS sensors, and also in the bandwidth and bandpass exo-atmospheric solar irradiance Eo among various IRS sensors.

The use of the simulation to combine hyperspectral and multispectral images was studied by Kruse (2009). Hyperspectral data were used to extract endmembers, which were then simulated at multispectral resolution. These endmembers were then used to extend the hyperspectral mapping to the larger spatial coverage offered by the multispectral data.

The spectral resolution requirements for mapping urban areas was investigated by Herold, Gardner, and Roberts (2003). They used AVIRIS data and an urban spectral library to study the most suitable spectral bands for separating urban land cover types. The AVIRIS data were also used to simulate Landsat TM and Ikonos data. The results showed that Ikonos and Landsat TM lack of spectral details to efficiently map several urban classes.

More recently, Masunaga et al. (2010) proposed a satellite data simulator unit which provides a tool to perform simulation. They also present applications of satellite simulation like model evaluation and algorithm development. Finally, Segl et al. (2010) also stressed the usefulness of remote sensing simulation for defining future Earth observation systems, optimizing instrument parameters, and developing and validating data-processing algorithms.

\subsection{Sensor simulation method}

To simulate multispectral data from hyperspectral data, the responses of narrow hyperspectral bands have to be aggregated. However, the reflectance values of the hyperspectral narrow bands cannot be summed directly to reproduce multispectral bands. Indeed, they must be weighted to account for the relative response of the multispectral bands. The RSR of each band of a sensor system is characterized by the effective spectral quantum efficiency, which indicates the spectral sensitivity of the band at each wavelength (Franke, Heinzel, and Menz 2006). Each sensor has 
consequently a different spectral sensitivity, which is described by its individual RSR functions. Figure 1 shows three examples of RSR functions for Quickbird, SPOT5-HRV and Landsat TM.

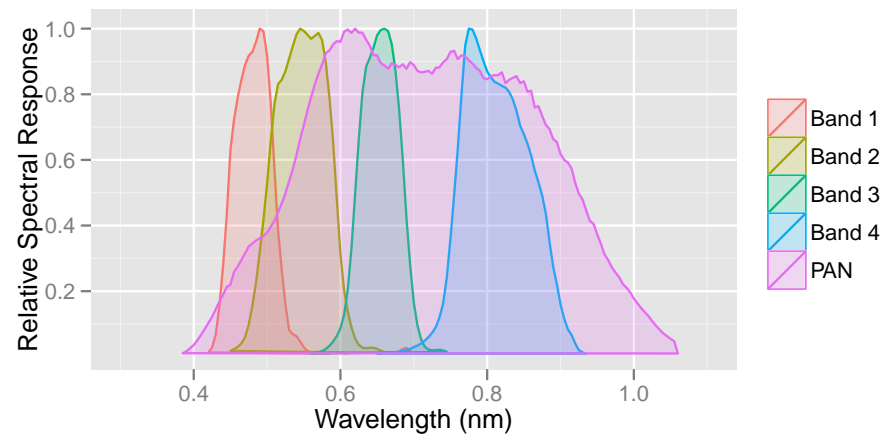

(a) Quickbird

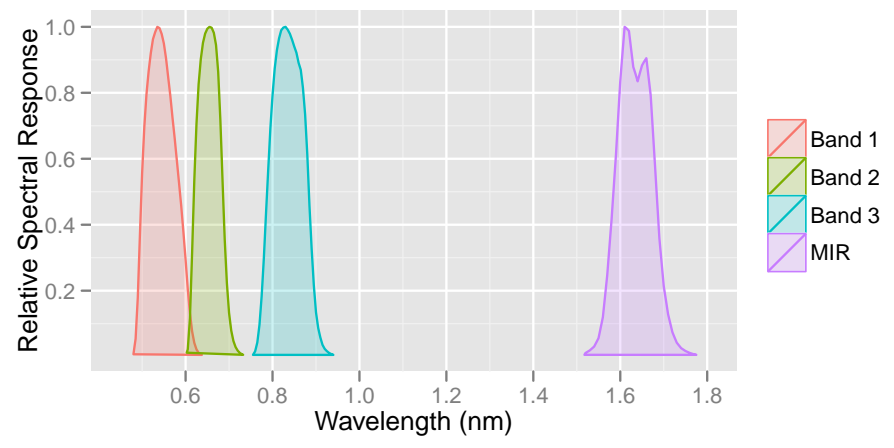

(b) SPOT5-HRV

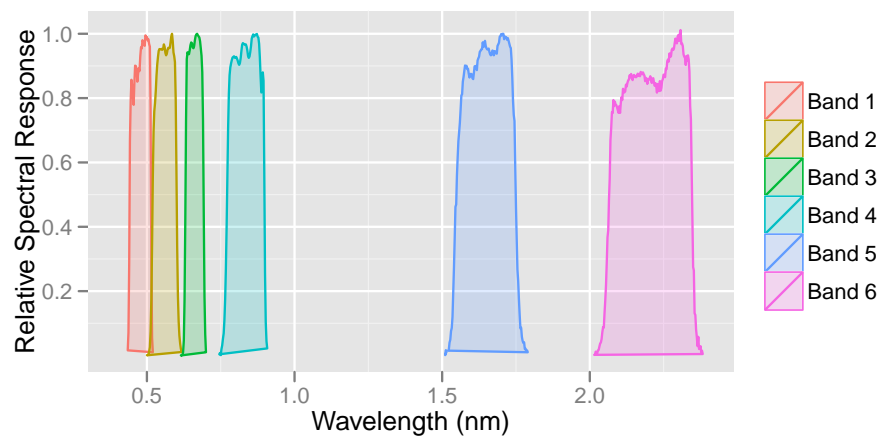

(c) Landsat TM

Figure 1. Example of RSR functions of three sensors namely, (a) Quickbird; (b) SPOT5-HRV and (c) Landsat TM.

As stated by Clark et al. (2002), different strategies have been proposed to compute the weights to apply to each hyperspectral band. For the simulation used in this paper, each hyperspectral center wavelength was linked with the mean RSR value (in the range of the full width half maximum (FWHM) of the hyperspectral spectral band) of the simulated band. This approach is similar to the one proposed by Franke, Heinzel, and Menz (2006). The equation of the simulation of the reflectance $L^{\text {sim }}$ according to the hyperspectral responses $L$ and the RSRs values $R$ for a band in the range $\left(b_{\min }, b_{\max }\right)$ can be expressed as follows: 


$$
L_{\left(b_{\min }, b_{\max }\right)}^{\operatorname{sim}}=\frac{\int_{b_{\min }}^{b_{\max }} L_{\lambda} R_{\lambda} \mathrm{d} \lambda}{\int_{b_{\min }}^{b_{\max }} R_{\lambda} \mathrm{d} \lambda}
$$

One should notice that some external parameters are not simulated in this study. For example, some other simulation approaches (Kavzoglu 2004) take into account other aspects like atmospheric effects or geometric differences between sensors. In this study, we are interested in the sensor discrimination ability according to their RSR, that is why we only focused on spectral differences caused by different RSR functions. Other aspects like the spatial resolution, or weather conditions of the acquisition (Rivard et al. 2011) should also be investigated and simulated to truly assess the differences between sensors. However, focusing only on spectral resolution already provides some insights on the different sensors ability.

Furthermore, we consider the used hyperspectral data as pure. Consequently, we did not apply other corrections (atmospheric corrections for example). As all simulations are made from the same spectra, the only variation in the simulation is the RSR functions of the sensors. This unique parameter variation approach allows us to fairly compare the different sensors.

\section{Spectral librairies}

\subsection{Available spectral libraries}

The advent of spectroscopy and remote sensing has offered an opportunity to develop a new kind of stored knowledge through spectral libraries. These spectral libraries are repositories of spectra of various kinds of materials (e.g. mineral, manmade material, vegetation, etc.) generally captured in situ using field instruments. Everybody agrees on the importance of the challenging problem to create such libraries in order to store, share and reuse information about materials. However, the number of freely available and easily accessible libraries is relatively limited. Indeed, an important number of factors limits their development, as the important cost of field acquisitions or the time needed to structure and organize the spectra in a meaningful way. In this section, we review the major available spectral libraries and their characteristics.

The most common and probably the most widely used library is the ASTER spectral library (Baldridge et al. 2008). This library includes contributions from the Jet Propulsion Laboratory (JPL), Johns Hopkins University (JHU) and the United States Geological Survey (USGS). It includes spectra of rocks, minerals, lunar soils, terrestrial soils, man-made materials, meteorites, vegetation, snow and ice covering the visible through thermal infrared wavelength region $(0.4-15.4 \mu \mathrm{m})$. The first version was released in July 1998 and the second one is available since 2007 on simple request through the library website ${ }^{1}$.

The USGS also offers its own library (Clark et al. 2007) which is freely available for download ${ }^{2}$. Researchers at the USGS Spectroscopy Lab have measured the spectral reflectance of hundreds of materials in the lab and have compiled a spectral

\footnotetext{
${ }^{1}$ http://speclib.jpl.nasa.gov

${ }^{2}$ http://speclab.cr.usgs.gov
} 
Table 1. Spectral libraries used in this study.

\begin{tabular}{lccccc}
\hline & \# Classes for each level & \# Spectra & \# Levels & Instrument & Wavelength range \\
\hline ASTER/JHU & $3 / 5 / 12$ & 270 & 3 & Beckman/Nicolet & {$[0.4 ; 14.0] \mu \mathrm{m}$} \\
ASTER/JPL & $6 / 20$ & 283 & 2 & Beckman/Nicolet & {$[14 ; 0.4] \mu \mathrm{m}$} \\
USGS & 4 & 860 & 1 & Beckman/ASD/Nicolet & {$[0.35 ; 2.5] \mu \mathrm{m}$} \\
NCGIA & 7 & 133 & 1 & AVIRIS & {$[0.37 ; 2.5] \mu \mathrm{m}$} \\
HEROLD & $4 / 8 / 19 / 26$ & 956 & 4 & AVIRIS & {$[0.37 ; 2.5] \mu \mathrm{m}$} \\
\hline
\end{tabular}

library. This library contains over 1300 spectra organized in six groups: minerals, soils, coatings, liquid, man-made and plants. To the best of our knowledge these two libraries (ASTER and USGS) are the most comprehensive and freely available libraries.

Other smaller projects provide sometimes spectra, as for example the National Center for Geographic Information and Analysis (NCGIA) which offers a small library of urban spectra. The spectral library developed for this project contains over 130 averaged spectra from AVIRIS sensor structured in 7 urban classes. The library is available for download on the project website ${ }^{3}$. Herold et al. (2004) also created a library of more than 4500 individual urban spectra from the city of Santa Barbara categorized in 108 unique surface types. Another library of 270 spectra from AVIRIS data was also developed.

Several attempts to create platforms to store and to share spectra also exist. For example, Hueni et al. (2009) proposed a software named $\mathrm{SPECCHIO}^{4}$, which is a tool to hold and structure reference spectra using a database. The authors make the distinction between spectral library (i.e. spectra list) and spectral database (i.e structured spectra with metadata). An interesting reflexion is discussed on the need to use standards and metadata.

Ferwerda, Jones, and Reston (2006) also offer a similar system through a website ${ }^{5}$ where researchers can store and share their spectra. The German aerospace center (DRL) also provides a spectral archive website ${ }^{6}$ with the intention to create a universal tool for archiving, managing and using spectra collected from various projects. Although these attempts to offer ways to share and store spectra are very interesting, they generally offer tools to structure the spectra but only provide few of them. Furthermore, the multiplication of these tools tends to bring confusion to the user interested in creating and managing a spectral library. It is worth noticing that all these different libraries are structured following their own format, which increases the difficulty to gather information coming from various sources.

\subsection{Spectral libraries used in this study}

In this study, four different libraries were used to assess the discrimination ability of the different sensors. Table 1 summarizes the specifications of the different libraries and the description of each library is given hereafter.

- ASTER/JHU : We chose to use the spectra from the JHU of the ASTER spectral library as they offer an interesting class hierarchy as well as useful wavelength range for spectra simulation.

- USGS : We used the spectra from USGS library which are defined in the $[0.35 ; 2.5] \mu \mathrm{m}$ range. Indeed, a certain number of spectra, especially mineral

\footnotetext{
${ }^{3}$ http://ncgia.ucsb.edu

${ }^{4}$ http://www.specchio.ch

${ }^{5}$ http://www . hyperspectral.info

${ }^{6}$ http://www. cocoon.caf.dlr.de
} 
ones, are defined in the thermal channel, which are not used for most of the multispectral sensors.

- NCGIA : We used all the available spectra.

- HEROLD This spectral library contains around 1000 spectra of urban materials structured in four different levels. This library comes from an AVIRIS flight over the Santa Barbara city in 2001. This library has already been used in (Herold, Gardner, and Roberts 2003; Herold et al. 2004) to study the spectral requirement for mapping urban areas.

\section{Evaluation}

\subsection{Class separability evaluation}

Separability criteria aim at evaluating the separability of the classes of a dataset in a given feature space (Davis and Swain 1978). Different criteria have been proposed in the literature to evaluate and quantify this separability. For remote sensing applications, the divergence criterion (D), the transformed divergence criterion (TD), the Bhattacharyya distance (B) and the Jeffreys-Matusita distance (JM) are the most widespread criteria. These criteria have been mainly used in feature selection problems, where the goal is to select the best subset of 'informative' or 'relevant' features for a given dataset, in order to maximize classification accuracy for this reduced number of features.

In multispectral and hyperspectral image classification, these criteria were used to select the best subset of bands (De Backer et al. 2005; Mi-Hyun and K. 2008; Riedmann and Milton 2003). Indeed, some bands may be noisy or correlated, and consequently provide a misleading information about class separability. Ranson et al. (2003) also used the JM distance to compare the separability of the same thematic classes in two different images, an optical one and a radar one. This study was carried out to choose which image was the best suited to identify these classes. Assuming a Gaussian distribution, these criteria use the mean and the covariance matrix of the classes. Their definitions are given hereafter.

Divergence:

$$
\begin{aligned}
D_{(i, j)}= & \frac{1}{2} \operatorname{tr}\left[\left(\Sigma_{i}-\Sigma_{j}\right)\left(\Sigma_{i}^{-1}-\Sigma_{j}^{-1}\right)\right] \\
& +\frac{1}{2} \operatorname{tr}\left[\left(\Sigma_{i}^{-1}-\Sigma_{j}^{-1}\right)\left(\mu_{i}-\mu_{j}\right)\left(\mu_{i}-\mu_{j}\right)^{T}\right]
\end{aligned}
$$

with $\mu_{i}$ and $\mu_{j}$ the means of the classes $i$ and $j, \Sigma_{i}$ and $\Sigma_{j}$ the covariance matrices and $\operatorname{tr}[$.$] the trace of the matrix.$

In the definition of the divergence (Eq.2) the second term on the right-hand side will increase continuously with the standard distance between the means of the pair of classes, thus the divergence will increase continuously with the standard distance between the means of the pair of classes. To solve this problem, a negative exponential term was introduced to transform the divergence (Bo, Wang, and Li 2005). Thus, the transformed divergence (TD) is defined as a saturated version of the divergence.

Transformed divergence:

$$
T D_{(i, j)}=2\left(1-e^{-\frac{1}{8} D_{(i, j)}}\right)
$$

The transformed divergence ranges in $[0 ; 2]$. Values in $[0.0 ; 1.0]$ indicate a very 
poor class separability, in $[1.0 ; 1.9]$ a poor separability and in $[1.9 ; 2.0]$ a relatively good separability.

Bhattacharyya distance:

$$
\begin{aligned}
B_{(i, j)}= & \frac{1}{2}\left[\mu_{i}-\mu_{j}\right]^{T}\left[\frac{\Sigma_{i}+\Sigma_{j}}{2}\right]^{-1}\left[\mu_{i}-\mu_{j}\right] \\
& +\frac{1}{2} \ln \frac{\left|\frac{1}{2}\left[\Sigma_{i}+\Sigma_{j}\right]\right|}{\sqrt{\left|\Sigma_{i}\right|\left|\Sigma_{j}\right|}}
\end{aligned}
$$

The Bhattacharyya distance, as the divergence, is unbounded. To cope with this problem, the Bhattacharyya distance is used in the Jeffreys-Matusita distance with a negative exponential term.

\section{Jeffreys-Matusita distance:}

$$
J M_{(i, j)}=\sqrt{2\left(1-e^{\left.-B_{(i, j)}\right)}\right.}
$$

The Jeffreys-Matusita distance ranges in $[0 ; 2]$ and the ranges of interpretation are barely similar to those of the transformed divergence.

These different criteria are designed to evaluate the separability between a couple of classes. To extend those to multiclass problems, different approaches have been proposed (Bruzzone, Roli, and Serpico 1995; Bruzzone and Serpico 2000). The most common strategy is to use the average or the weighted average distances computed for all pairs of classes.

$$
\begin{aligned}
& J M_{\text {avg }}=\frac{C(C-1)}{2} \sum_{i=1}^{C-1} \sum_{j=i+1}^{C} J M_{(i, j)} \\
& T D_{\text {avg }}=\frac{C(C-1)}{2} \sum_{i=1}^{C-1} \sum_{j=i+1}^{C} T D_{(i, j)}
\end{aligned}
$$

These means can be weighted to take into account the class prior probability $p(\omega)$ :

$$
\begin{aligned}
& J M_{\text {wavg }}=\sum_{i=1}^{C} \sum_{j>i}^{C} p\left(\omega_{i}\right) p\left(\omega_{j}\right) J M_{(i, j)} \\
& T D_{\text {wavg }}=\sum_{i=1}^{C} \sum_{j>i}^{C} p\left(\omega_{i}\right) p\left(\omega_{j}\right) T D_{(i, j)}
\end{aligned}
$$

Another formulation, which better approximates the Bayes error using JeffreysMatusita distance, is given by Bruzzone and Serpico (2000):

$$
J M_{\text {wavg }}=\sum_{i=1}^{C} \sum_{j>i}^{C} \sqrt{p\left(\omega_{i}\right) p\left(\omega_{j}\right)} J M_{(i, j)}^{2}
$$




$$
T D_{w a v g}=\sum_{i=1}^{C} \sum_{j=1}^{C} p\left(\omega_{i}\right) p\left(\omega_{j}\right) T D_{(i, j)}
$$

Alternatively, the minimum Jeffreys-Matusita distance is sometimes used to evaluate the minimum distance between a couple of classes among a set of classes. It evaluates the minimum separability among the classes and can be used as an indicator of separability of the whole dataset.

$$
J M_{\min }=\min \left(J M_{(i, j)}\right) \quad \forall i, j \in[1 \ldots C] \quad i \neq j
$$

An intensive work is still under progress, mainly in the fields of pattern recognition and data mining, to propose new criteria to solve the features selection problem. Recent works use information theory and subspace analysis to design new criteria (Guo et al. 2008; Lai, Reinders, and Wessels 2006; Sotoca, Pla, and Sanchez 2007; Gunala and Edizkanb 2008; Liua et al. 2009). However, the criteria presented in this section are the most commonly used, especially in the remote sensing field. An exhaustive presentation of all criteria is out of scope of this paper.

The choice between Jeffreys-Matusita distance and the divergence to evaluate the separability of a set of classes is not trivial. Richards and Jia (2006) claim that Jeffreys-Matusita distance tends to perform better as a feature selection criterion than the divergence, but is computationally more complex. This is mainly due to the high number of matrix operations required to compute the Bhattacharyya distance. The Jeffreys-Matusita distance and the transformed divergence are described as almost as effective and considerably better than simple divergence and simple Bhattacharyya, essentially thanks to their bounded range. The Jeffreys-Matusita distance and the transformed divergence seem equally used in the literature without clear arguments on why choosing one or the other. In this paper we chose to use the Jeffreys-Matusita distance. Recent studies (Gunal and Edizkan 2008; Maghsoudi, Zoej, and Collins 2011) mainly focuse on subspace based feature selection and reduce the dimensionality problems.

\subsection{Classification accuracy}

Another way of evaluating class separability and assessing features relevance is to perform a supervised classification of the dataset. The accuracy and the confusion matrix of the classification are useful tools to assess the quality of features. Indeed, as stated by Heidena et al. (2007), separability criteria can suffer from some problems in extreme cases. For example, a class can show a good separability with the other classes with a constant overlap of $5 \%$ for each class in the feature space. However, the total spectral overlap can range from $5 \%$ allowing a classification of good quality to $100 \%$ making the accurate classification of this material impossible. Furthermore, the computation of the Bhattacharyya distance requires the calculation of the inverse of the covariance matrix, which can be sometimes problematic. Indeed, when the number of features increases, the covariance matrix tends to become singular and consequently not invertible.

To evaluate the classification performance on the spectral libraries we used an ensemble classifier system (Kittler et al. 1998), which combines three different supervised methods: a 1-Nearest-Neighbour classifier, a Naive Bayes classifier, and 
Table 2. Information about the different sensors included in this study.

\begin{tabular}{|c|c|c|c|c|}
\hline Name & \# Bands & \# Owner & Bands $(\mu \mathrm{m})$ & Resolution \\
\hline Spot 5-HRV & 4 & CNES & $\begin{array}{l}{[0.50 ; 0.59][0.61 ; 0.68][0.78 ;} \\
0.89][1.58 ; 1.75] \\
{[0.51 ; 0.73]}\end{array}$ & $\begin{array}{l}10 \times 10 \mathrm{~m} \\
2.5 \times 2.5 \mathrm{~m} \\
\end{array}$ \\
\hline Quickbird & 5 & Digital Globe & $\begin{array}{l}{[0.45 ; 0.52][0.52 ; 0.60]} \\
0.69][0.76 ; 0.90] \\
{[0.445 ; 0.900]}\end{array}$ & $\begin{array}{l}2.44 \times 2.44 \mathrm{~m} \\
0.61 \times 0.61 \mathrm{~m}\end{array}$ \\
\hline Pleiades & 5 & CNES & $\begin{array}{l}{[0.43 ; 0.55][0.49 ; 0.61][0.60 ;} \\
0.72][0.75 ; 0.90] \\
{[0.445 ; 0.900]}\end{array}$ & $\begin{array}{c}0.7 \times 0.7 \mathrm{~m} \\
0.61 \times 0.61 \mathrm{~m} \\
\end{array}$ \\
\hline Landsat TM & 6 & NASA & $\begin{array}{lllll}{[0.45 ;} & 0.52] & {[0.52 ;} & 0.60] & {[0.63 ;} \\
0.69] & {[0.76 ;} & 0.90] & {[1.55 ;} & 1.75] \\
{[2.08 ;} & 2.35] & & & \\
{[10.4 ; 12.5]} & & & \end{array}$ & $\begin{array}{c}30 \times 30 \mathrm{~m} \\
120 \times 120 \mathrm{~m}\end{array}$ \\
\hline Ikonos & 4 & $\mathrm{SIC}$ & $\begin{array}{l}{[0.45 ; 0.52][0.52 ; 0.60]} \\
0.69][0.76 ; 0.90] \\
{[0.45 ; 0.90]}\end{array}$ & $\begin{array}{l}4 \times 4 \mathrm{~m} \\
1 \times 1 \mathrm{~m} \\
\end{array}$ \\
\hline Formosat-2 & 5 & NSPO & $\begin{array}{l}{[0.45 ; 0.52][0.52 ; 0.60][0.63 ;} \\
0.69][0.76 ; 0.90] \\
{[0.45 ; 0.90]}\end{array}$ & $\begin{array}{l}8 \times 8 \mathrm{~m} \\
2 \times 2 \mathrm{~m}\end{array}$ \\
\hline Meris & 15 & ESA & $\begin{array}{l}{[0.39 ; 1.04] \text { bandwidth }} \\
\text { grammable between } 2.5 \text { and } \\
30 \mathrm{~nm}\end{array}$ & $1000 \times 1000 \mathrm{~m}$ \\
\hline
\end{tabular}

a C4.5 classifier. These classifiers were combined through a majority voting strategy. This choice has been made to reduce the bias involved in the selection of a single algorithm. For the experiment, 10-cross-validation has been used. It consists in splitting the dataset in 10 subsets and then, in learning on $\frac{9}{10}$ of the dataset and then evaluating on the remaining $\frac{1}{10}$. The evaluation is computed for all the combinations. This approach avoids to split the dataset in a learning set and an evaluation set and is statistically more relevant (as each sample is used alternatively for learning and testing).

\section{Experiments}

In this section, we present three different experiments carried out to illustrate the usefulness of sensor simulation. Table 2 summarizes the information on the sensors used in the experiments, which are some of the most common multispectral systems available.

In the first experiment (Section 5.1), we compared the different sensors according to their spectral response defined by their RSR function. The different libraries presented in Section 3 were convolved with the RSR of each sensor. Then, separability index and classification accuracy were computed in order to compare the different sensors. Hence, we were able to gain some insight on the sensor ability to classify materials accurately. Furthermore, the comparison is fair as the dataset for each sensor is created from the exact same spectral libraries. The only variation for each dataset is the RSR used to convolve the spectra.

The second experiment (Section 5.2), consisted in evaluating the potential interest of spectral indexes (e.g., NDVI). The aim of this simulation was to evaluate if a specific spectral index was useful for the classification of a specific material, and if the sensors were able to leverage this new information. Classification accuracy assessment and separability evaluation were conducted with and without the use of spectral indexes in order to evaluate the interest of their addition to the dataset.

The last experiment (Section 5.3) illustrates one of the many potential uses of sensor simulation. In this experiment, we evaluated the interest of a specific band, 
Table 3. Sensors comparison on all the available datasets.

\begin{tabular}{|c|c|c|c|c|c|c|c|c|c|c|c|c|c|c|}
\hline \multirow{2}{*}{ Library } & \multicolumn{2}{|c|}{ Ikonos } & \multicolumn{2}{|c|}{ Pleiades } & \multicolumn{2}{|c|}{ Quickbird } & \multicolumn{2}{|c|}{ Formosat-2 } & \multicolumn{2}{|c|}{ Landsat } & \multicolumn{2}{|c|}{ Spot 5-HRV } & \multicolumn{2}{|c|}{ Meris } \\
\hline & JM & Vote & JM & Vote & JM & Vote & $\mathrm{JM}$ & Vote & $\mathrm{JM}$ & Vote & $\mathrm{JM}$ & Vote & JM & Vote \\
\hline Aster I & .79 & 83.3 & 0.82 & 92.2 & 0.76 & 84.4 & 0.76 & 84.8 & 0.85 & 94.4 & 0.76 & 89.2 & 0.92 & 94.4 \\
\hline Aster II & 1.20 & 53.3 & 1.21 & 61.1 & 1.23 & 51.4 & 1.16 & 51.1 & 1.29 & 62.5 & 1.16 & 57.7 & 1.55 & 64.8 \\
\hline Aster III & 1.31 & 45.1 & 1.29 & 50.0 & 1.31 & 44.8 & 1.28 & 45.9 & 1.33 & 48.8 & 1.26 & 50.7 & 1.60 & 51.8 \\
\hline NCGIA & 1.38 & 59.3 & 1.38 & 62.4 & 1.39 & 61.6 & 1.38 & 60.1 & 1.41 & 60.9 & 1.37 & 63.1 & 0.76 & 56.8 \\
\hline USGS & 0.84 & 77.0 & 0.86 & 77.6 & 0.84 & 75.9 & 0.84 & 76.4 & 0.92 & 79.5 & 0.86 & 77.5 & 1.21 & 79.5 \\
\hline Herold I & 0.79 & 96.4 & 0.78 & 96.5 & 0.78 & 96.4 & 0.78 & 96.8 & 0.81 & 97.8 & 0.78 & 96.7 & 0.75 & 97.2 \\
\hline Herold II & 1.28 & 91.5 & 1.26 & 91.8 & 1.30 & 90.5 & 1.23 & 91.7 & 1.31 & 93.3 & 1.29 & 91.6 & 0.89 & 92.2 \\
\hline Herold III & 1.76 & 85.6 & 1.74 & 86.6 & 1.76 & 85.1 & 1.75 & 85.3 & 1.80 & 91.4 & 1.78 & 88.1 & 1.55 & 88.3 \\
\hline Herold IV & 1.84 & 85.3 & 1.81 & 86.7 & 1.84 & 85.2 & 1.82 & 85.1 & 1.85 & 90.6 & 1.84 & 88.3 & 1.75 & 87.4 \\
\hline
\end{tabular}

namely the Short-Wave Infra-Red band (SWIR). To study the usefulness of this band, we carried out two evaluations: the first one consisted in removing the SWIR band from a sensor equipped of this band, when the second one consisted in adding this band to a sensor not originally equipped. This experiment reveals how sensor simulation can be used to design new sensors and evaluate the interest of a specific spectral band.

\subsection{Sensors evaluation and comparison}

In this first experiment the different libraries were used to compare the different sensors. The results are presented in Table 3 where the accuracy of the ensemble classifier is presented in the column Vote, and the Jeffreys-Matusita distance in the column JM. We discuss the results for each dataset hereafter.

ASTER : The results for this data set are presented in Table 4. The Ikonos and Formosat-2 sensors give the lowest results. This is consistent as they have a low spectral resolution compared to the other studied sensors. They are followed by Spot 5-HRV, Quickbird and Pleiades and finally Meris which achieves the best results. An interesting point to notice in these results is the relative large difference between the accuracy of Quickbird (84.4\%) and Pleiades (92.2\%). As these two sensors have the same number of bands which are very similar, one could expect a similar behavior in classification accuracy. However, even if the RSR are strongly similar, there are still some small variations. The most important difference in the bands of these two sensors is the Near Infra Red (NIR) band. To check if the difference of accuracy was due to this difference of RSR, we removed the NIR band of these two sensors and ran the classification again. This time, the accuracies were quite close with $83.3 \%$ for Pleiades and $81.5 \%$ for Quickbird. Then, we replaced the NIR band of Quickbird by the NIR band of Pleiades to check if the NIR band of Pleiades was truly responsible of the higher accuracy. We obtained this time 92.2\% for Pleiades and $91.1 \%$ for Quickbird (instead of $84.4 \%$ with its own NIR band). These results supported our hypothesis that the variation in the NIR band was responsible of the difference of accuracy.

We investigated more closely the results to identify some examples where Pleiades was able to correctly discriminate two specific spectra, while Quickbird was not. We identified several couples of spectra misclassified by Quickbird and well classified by Pleiades. We present here an example with an Asphalt spectrum (Man-Made) and a Basalt spectrum (Rocks) (see Figure 2 (a)). On Figure 2 (b), one can observe that the NIR bands of the two sensors are quite similar, but not exactly defined in the same range of wavelengths. This small shift on the wavelength seems to be responsible of the difference of accuracy. On Figure 2 (c), one can clearly see that the two spectra are very close in the range of the Quickbird NIR band while they 
Table 4. Results of sensor evaluation and comparison for the ASTER/JHU data set.

\begin{tabular}{|c|c|c|c|c|c|c|c|c|c|c|}
\hline Sensor & Level & $J M_{\text {wave }}$ & $J M_{a v e}$ & $J M_{\min }$ & $T D_{\text {wave }}$ & $T D_{\text {ave }}$ & $1 \mathrm{NN}$ & NaiveBayes & $\mathrm{J} 48$ & Vote \\
\hline Ikonos & I & 0.796 & 1.780 & 1.531 & 0.884 & 1.922 & 82.963 & 58.519 & 80.370 & 83.333 \\
\hline- & II & 1.204 & 1.584 & 0.938 & 1.394 & 1.780 & 53.333 & 41.852 & 48.889 & 53.333 \\
\hline- & III & 1.312 & 1.836 & 0.320 & 1.495 & 1.976 & 44.074 & 32.963 & 37.407 & 45.185 \\
\hline Pleiades & I & 0.825 & 1.825 & 1.599 & 0.878 & 1.912 & 92.593 & 62.593 & 86.296 & 92.222 \\
\hline- & II & 1.212 & 1.597 & 0.978 & 1.341 & 1.726 & 60.741 & 46.296 & 54.074 & 61.111 \\
\hline- & III & 1.293 & 1.772 & 0.128 & 1.447 & 1.956 & 47.778 & 35.185 & 42.222 & 50.000 \\
\hline Quickbird & I & 0.761 & 1.729 & 1.540 & 0.884 & 1.921 & 83.704 & 59.259 & 81.111 & 84.444 \\
\hline- & II & 1.237 & 1.616 & 1.004 & 1.455 & 1.858 & 52.593 & 41.852 & 47.037 & 51.481 \\
\hline- & III & 1.314 & 1.765 & 0.446 & 1.546 & 1.985 & 42.222 & 32.593 & 37.037 & 44.815 \\
\hline Formosat-2 & $\mathrm{I}$ & 0.763 & 1.696 & 1.605 & 0.864 & 1.886 & 85.926 & 58.519 & 80.000 & 84.815 \\
\hline- & II & 1.169 & 1.524 & 1.034 & 1.350 & 1.725 & 53.333 & 42.593 & 48.519 & 51.111 \\
\hline- & III & 1.281 & 1.718 & 0.386 & 1.471 & 1.968 & 45.185 & 32.963 & 37.037 & 45.926 \\
\hline Landsat & $\mathrm{I}$ & 0.858 & 1.882 & 1.721 & 0.926 & 1.994 & 94.815 & 85.556 & 91.852 & 94.444 \\
\hline- & II & 1.296 & 1.698 & 0.928 & 1.469 & 1.887 & 62.222 & 57.778 & 60.000 & 62.593 \\
\hline- & III & 1.335 & 1.732 & 0.370 & 1.537 & 1.984 & 49.259 & 41.481 & 37.778 & 48.889 \\
\hline Spot 5-HRV & $\mathrm{I}$ & 0.761 & 1.727 & 1.504 & 0.901 & 1.951 & 90.000 & 71.481 & 84.074 & 89.259 \\
\hline- & II & 1.161 & 1.529 & 0.760 & 1.348 & 1.747 & 60.000 & 50.370 & 50.370 & 57.778 \\
\hline- & III & 1.268 & 1.747 & 0.308 & 1.433 & 1.949 & 48.148 & 35.926 & 43.704 & 50.741 \\
\hline Meris & $\mathrm{I}$ & 0.924 & 1.992 & 1.975 & 0.929 & 2.000 & 94.444 & 60.000 & 88.889 & 94.444 \\
\hline- & II & 1.557 & 1.982 & 1.925 & 1.572 & 2.000 & 64.815 & 50.370 & 58.519 & 64.815 \\
\hline- & III & 1.602 & 1.943 & 1.169 & 1.636 & 2.000 & 52.963 & 35.926 & 43.333 & 51.852 \\
\hline
\end{tabular}

Table 5. Results of sensor evaluation and comparison for the USGS data set.

\begin{tabular}{lccccccccc}
\hline Sensor & $J M_{\text {wave }}$ & $J M_{\text {ave }}$ & $J M_{\min }$ & $T D_{\text {wave }}$ & $T D_{\text {ave }}$ & 1NN & NaiveBayes & J48 & Vote \\
\hline Ikonos & 0.840 & 1.309 & 0.706 & 1.063 & 1.709 & 73.140 & 57.791 & 74.302 & 77.093 \\
Pleiades & 0.869 & 1.377 & 0.768 & 1.073 & 1.756 & 74.503 & 59.883 & 76.959 & 77.661 \\
Quickbird & 0.842 & 1.317 & 0.738 & 1.061 & 1.721 & 73.372 & 58.023 & 73.488 & 75.930 \\
Formosat-2 & 0.849 & 1.353 & 0.651 & 1.059 & 1.744 & 73.216 & 59.649 & 74.269 & 76.491 \\
Landsat & 0.926 & 1.421 & 1.170 & 1.138 & 1.765 & 76.140 & 75.439 & 76.257 & 79.532 \\
Spot 5-HRV & 0.866 & 1.324 & 0.909 & 1.096 & 1.718 & 72.749 & 73.567 & 75.322 & 77.544 \\
Meris & 1.216 & 1.974 & 1.862 & 1.237 & 1.999 & 76.118 & 61.882 & 78.471 & 79.529 \\
\hline
\end{tabular}

seem to be more separable in the Pleiades NIR band range.

These results are consistent with previous studies which also pointed out that even small variations in band definition can imply differences in spectral response of some materials. For example, in Franke, Heinzel, and Menz (2006), the authors showed that the variation on the NIR band of different sensors leads to strong variation in the calculation of the NDVI. In Meyer and Chander (2007), the authors showed that the definition of the RSR is a key issue to allow the identification of some type of materials. The conclusion from these results is not that Pleiades is better than Quickbird, but rather than even two sensors having similar bands can produce different results according to the application. Furthermore, these differences might be estimated and evaluated thanks to sensors simulation as in the present study. Figure 3 presents the accuracy of each class at each level for each sensor.

USGS : The results for the USGS data set are consistent with the spectral resolution of the sensor (see Table 5). The best results are achieved by Landsat and Meris with an accuracy of $79.5 \%$. It seems that the Landsat bands are especially well suited for this data set and helps to better discriminate several spectra confused by the other sensors.

NCGIA : In this data set, the best results are achieved by SPOT 5-HRV sensor. The SWIR channel seems useful in this application to discriminate some urban land covers like Road and Roof, which are well known as difficult to classify. The Table 6 presents the results for the different sensors and the Figure 4 the accuracy for each class. 


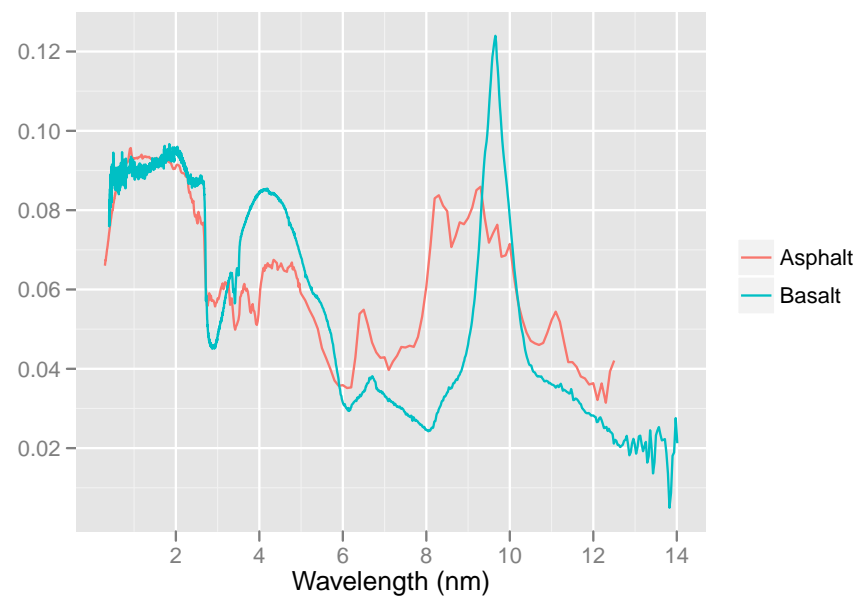

(a) Asphalt and Basalt spectra

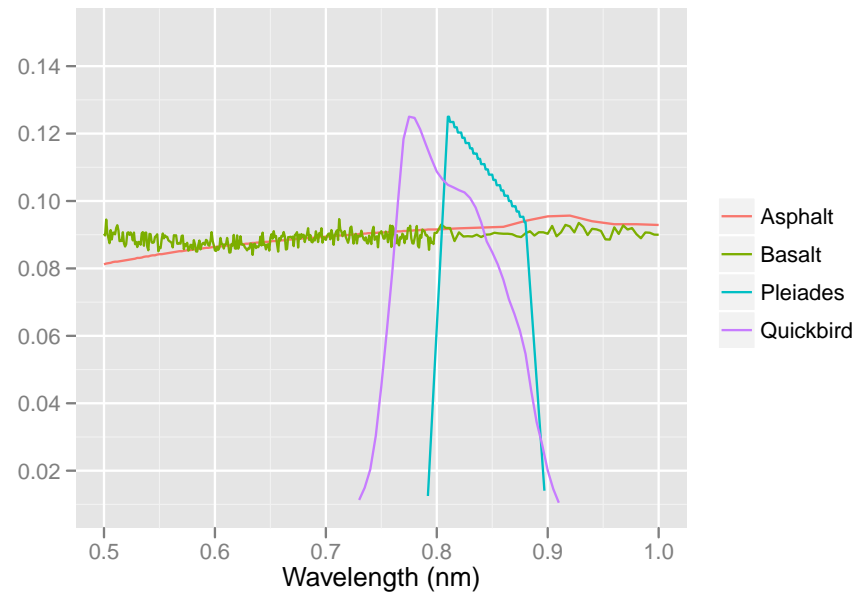

(b) Zoomed Asphalt and Basalt spectra and the NIR band of Quickbird and Pleiades

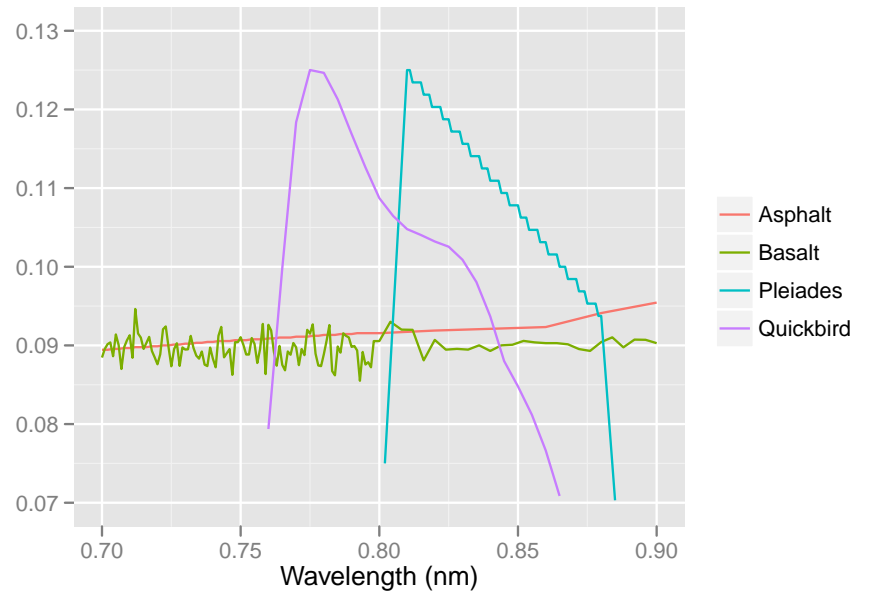

(c) Zoomed Asphalt and Basalt spectra and the NIR band of Quickbird and Pleiades

Figure 2. Asphalt (man-made materials) and basalt (rocks) spectra (a) and Zoomed Asphalt and Basalt spectra and the NIR bands of QuickBird and Pleiades (b, c). 


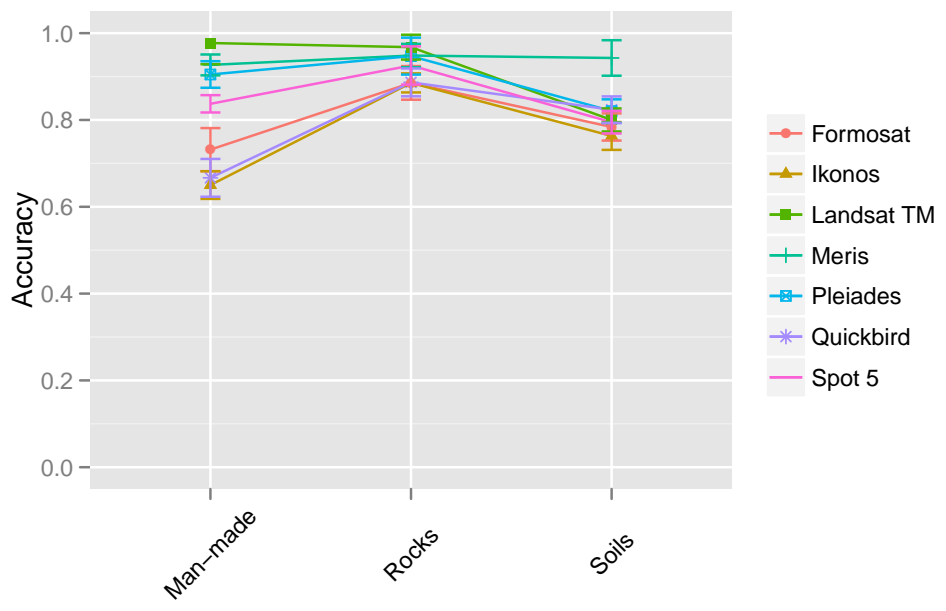

(a) Classification accuracy per class for ASTER/JHU Level 2

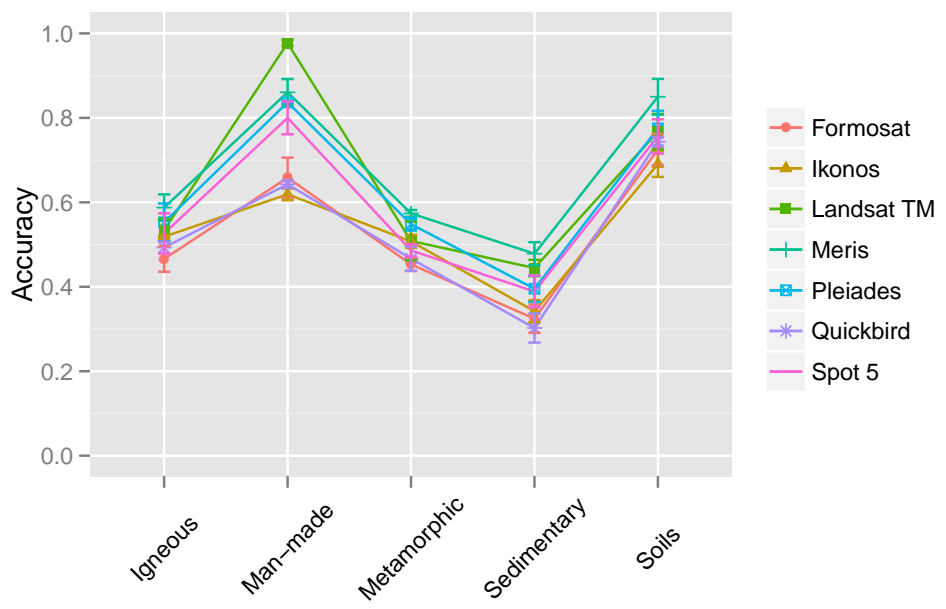

(b) Classification accuracy per class for USGS

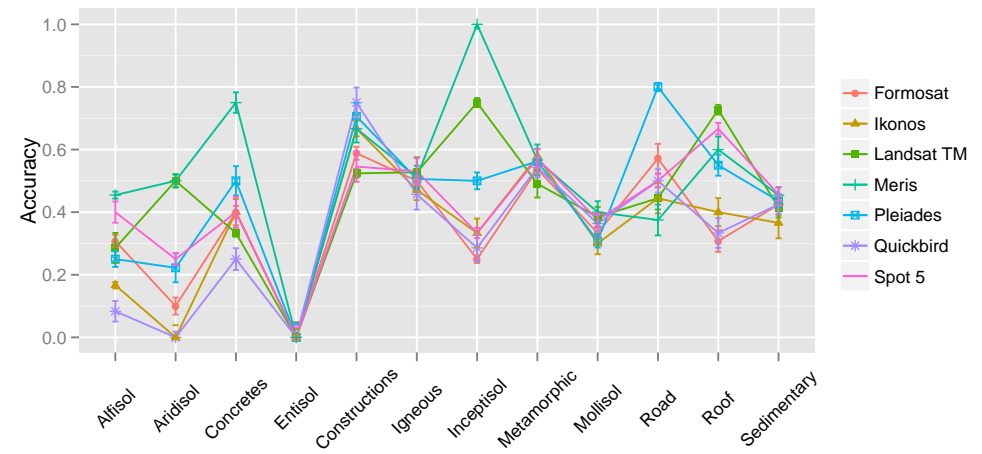

(c) Classification accuracy per class for USGS

Figure 3. Classification accuracy per class for ASTER/JHU Level I (a), Level II (b), and Level III (c). 


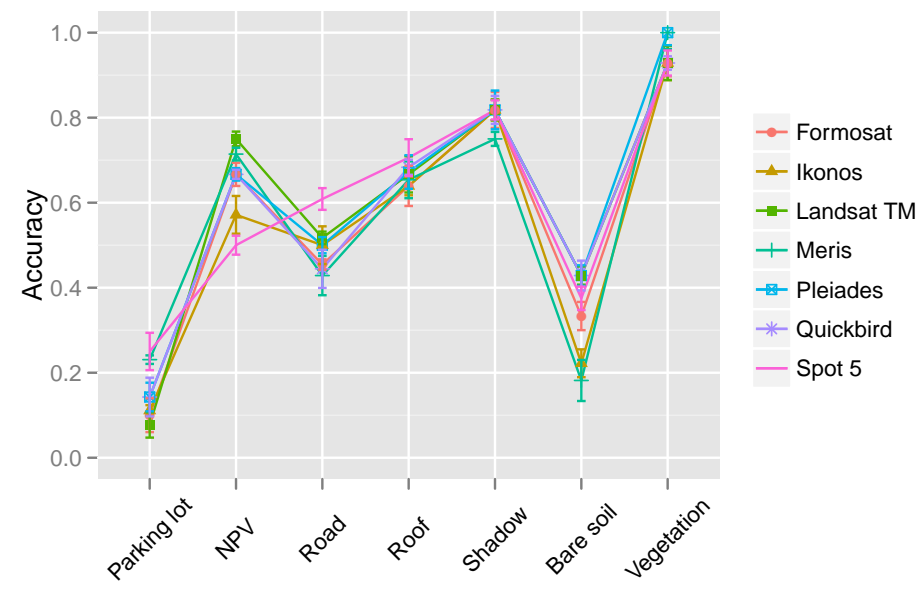

Figure 4. Classification accuracy per class for NCGIA

Table 6. Results of sensor evaluation and comparison for the NCGIA data set.

\begin{tabular}{lccccccccc}
\hline Sensor & $J M_{\text {wave }}$ & $J M_{\text {ave }}$ & $J M_{\min }$ & TD wave & TDave & 1NN & NaiveBayes & J48 & Vote \\
\hline Ikonos & 1.386 & 1.946 & 1.497 & 1.409 & 1.977 & 57.895 & 42.857 & 57.895 & 59.398 \\
Pleiades & 1.386 & 1.940 & 1.474 & 1.162 & 1.824 & 57.143 & 44.361 & 56.391 & 62.406 \\
Quickbird & 1.393 & 1.952 & 1.578 & 1.410 & 1.976 & 59.398 & 42.857 & 54.887 & 61.654 \\
Formosat & 1.382 & 1.942 & 1.444 & 1.338 & 1.939 & 56.391 & 42.857 & 54.887 & 60.150 \\
Landsat & 1.419 & 1.977 & 1.705 & 1.449 & 1.998 & 57.143 & 59.398 & 57.895 & 60.902 \\
Spot 5-HRV & 1.371 & 1.939 & 1.380 & 1.405 & 1.970 & 55.639 & 44.361 & 59.398 & 63.158 \\
Meris & 0.766 & 1.441 & 0.135 & 1.438 & 1.984 & 53.030 & 36.364 & 58.333 & 56.818 \\
\hline
\end{tabular}

Herold : One of the interesting things to observe in the results of this data set (Table 7), is the behavior of the sensors according to the level of classification. Indeed, this data set comes with a class hierarchy of four levels. The classification is expected to be more difficult as the number of classes increases, since the probability of confusion between the classes increases as well. In levels I and II, the results are barely similar among the different sensors with an accuracy around $96 \%$ for level I and $91 \%$ for level II. However, at level III, some differences of accuracy appear and again, the sensors with better spectral resolution have better results. Landsat has particularly good results with an accuracy of $91.4 \%$ followed by Meris with $88.3 \%$. The trend is maintained on level IV. This kind of experiment allows us to gain some insight on the ability of the sensors to map land cover at certain levels. If a user is interested in mapping some general classes (Level I and II), the choice of the sensor is not crucial. However, if the user is interested in mapping specific classes deeper in the hierarchy (Level III and IV), the user should carefully select a sensor which accurately maps these classes. 


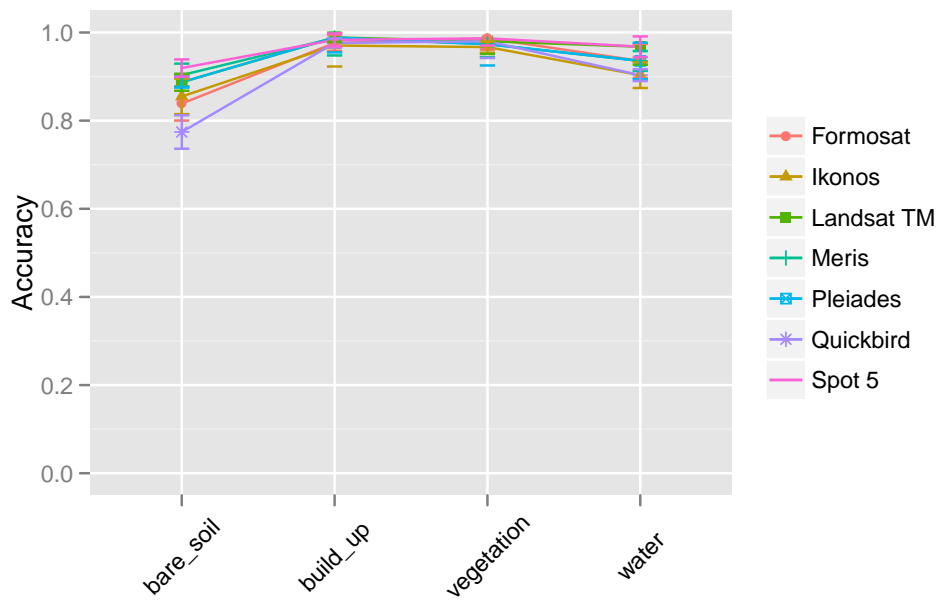

(a) Level 1

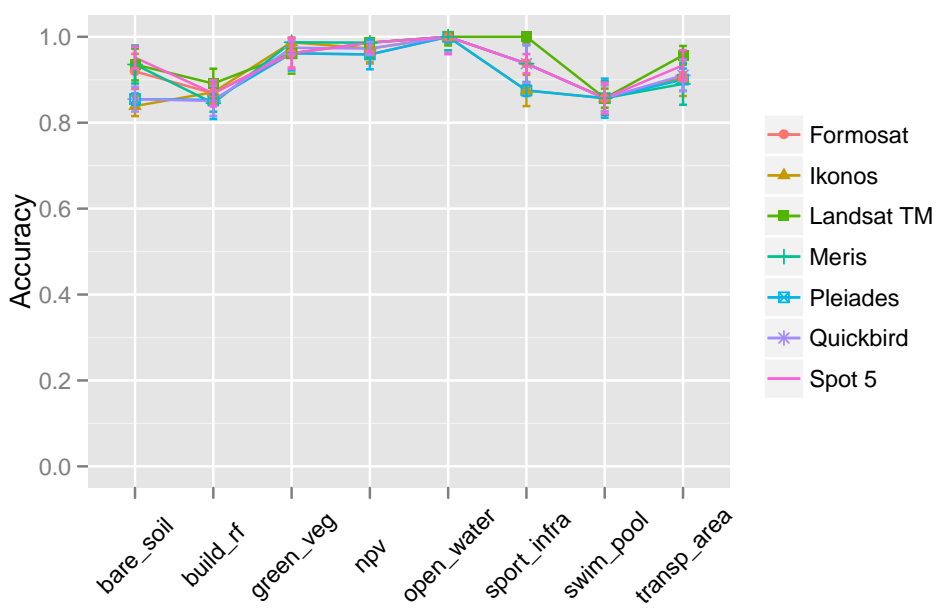

(b) Level 2

Figure 5. Classification accuracy per class for HEROLD Level 1 (a) and Level 2 (b)

Table 7. Results of sensor evaluation and comparison for the HEROLD data set.

\begin{tabular}{|c|c|c|c|c|c|c|c|c|c|c|}
\hline Sensor & Level & $J M_{\text {wave }}$ & $J M_{\text {ave }}$ & $J M_{\min }$ & $T D_{\text {wave }}$ & TD ave & $1 \mathrm{NN}$ & NaiveBayes & $\mathrm{J} 48$ & Vote \\
\hline \multirow{4}{*}{ Ikonos } & I & 0.790 & 1.920 & 1.819 & 0.827 & 1.994 & 97.490 & 64.958 & 94.456 & 96.444 \\
\hline & II & 1.284 & 1.966 & 1.512 & 1.327 & 1.985 & 93.410 & 56.590 & 87.866 & 91.527 \\
\hline & III & 1.763 & 1.949 & 1.067 & 1.769 & 1.954 & 87.552 & 59.414 & 80.544 & 85.669 \\
\hline & IV & 1.840 & 1.956 & 1.402 & 1.838 & 1.953 & 87.552 & 57.322 & 81.276 & 85.356 \\
\hline \multirow{4}{*}{$\overline{\text { Pleiades }}$} & I & 0.787 & 1.903 & 1.810 & 0.808 & 1.956 & 98.222 & 61.402 & 96.339 & 96.548 \\
\hline & II & 1.263 & 1.946 & 1.464 & 1.024 & 1.920 & 93.933 & 56.276 & 87.029 & 91.841 \\
\hline & III & 1.741 & 1.929 & 1.139 & 1.608 & 1.821 & 89.644 & 60.669 & 82.113 & 86.611 \\
\hline & IV & 1.810 & 1.924 & 1.238 & 1.695 & 1.771 & 89.121 & 59.937 & 81.590 & 86.715 \\
\hline \multirow[t]{4}{*}{ Quickbird } & $\mathrm{I}$ & 0.782 & 1.913 & 1.794 & 0.827 & 1.994 & 98.013 & 65.377 & 95.188 & 96.444 \\
\hline & II & 1.301 & 1.966 & 1.573 & 1.322 & 1.985 & 93.305 & 56.276 & 86.715 & 90.586 \\
\hline & III & 1.764 & 1.948 & 1.109 & 1.771 & 1.954 & 87.866 & 59.728 & 79.916 & 85.146 \\
\hline & IV & 1.840 & 1.958 & 1.350 & 1.839 & 1.954 & 87.552 & 58.264 & 80.962 & 85.251 \\
\hline \multirow[t]{4}{*}{ Formosat-2 } & $\mathrm{I}$ & 0.782 & 1.897 & 1.784 & 0.825 & 1.989 & 98.222 & 64.226 & 95.607 & 96.862 \\
\hline & II & 1.232 & 1.947 & 1.345 & 1.177 & 1.962 & 94.038 & 56.695 & 86.088 & 91.736 \\
\hline & III & 1.750 & 1.938 & 1.110 & 1.713 & 1.911 & 88.285 & 59.937 & 79.498 & 85.356 \\
\hline & IV & 1.828 & 1.946 & 1.304 & 1.796 & 1.902 & 87.866 & 58.473 & 80.858 & 85.146 \\
\hline \multirow[t]{4}{*}{ Landsat } & $\mathrm{I}$ & 0.814 & 1.967 & 1.923 & 0.830 & 2.000 & 99.582 & 79.707 & 96.234 & 97.803 \\
\hline & II & 1.314 & 1.981 & 1.595 & 1.374 & 1.993 & 96.548 & 53.556 & 88.598 & 93.305 \\
\hline & III & 1.809 & 1.976 & 1.469 & 1.821 & 1.980 & 93.619 & 65.272 & 85.669 & 91.423 \\
\hline & IV & 1.856 & 1.963 & 0.546 & 1.859 & 1.976 & 92.887 & 69.979 & 87.762 & 90.690 \\
\hline \multirow{4}{*}{ Spot 5-HRV } & $\mathrm{I}$ & 0.789 & 1.907 & 1.784 & 0.830 & 2.000 & 98.745 & 79.707 & 96.025 & 96.757 \\
\hline & II & 1.291 & 1.964 & 1.549 & 1.317 & 1.985 & 94.979 & 57.008 & 86.402 & 91.632 \\
\hline & III & 1.782 & 1.956 & 1.153 & 1.803 & 1.967 & 91.423 & 63.075 & 82.531 & 88.180 \\
\hline & IV & 1.841 & 1.961 & 0.950 & 1.849 & 1.960 & 90.377 & 65.481 & 82.741 & 88.389 \\
\hline \multirow[t]{4}{*}{ Meris } & I & 0.756 & 1.719 & 1.163 & 0.830 & 1.999 & 98.222 & 65.481 & 97.280 & 97.280 \\
\hline & II & 0.896 & 1.839 & 0.179 & 11647 & 1.989 & 94.142 & 54.289 & 87.657 & 92.259 \\
\hline & III & 1.553 & 1.802 & 0.346 & 1.711 & 1.927 & 89.749 & 58.473 & 83.787 & 88.389 \\
\hline & IV & 1.754 & 1.846 & 0.246 & 1.813 & 1.925 & 89.121 & 59.728 & 83.264 & 87.448 \\
\hline
\end{tabular}




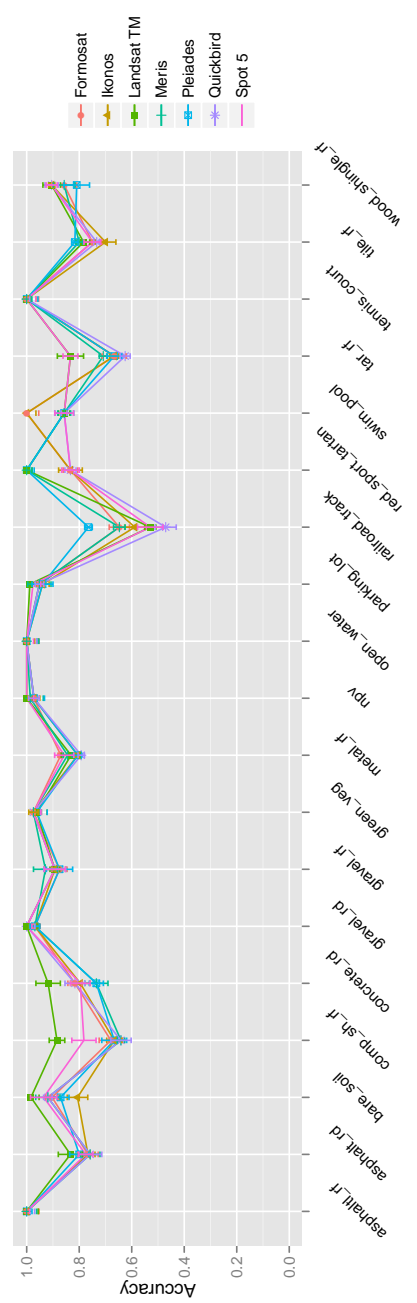

(a) Level 3

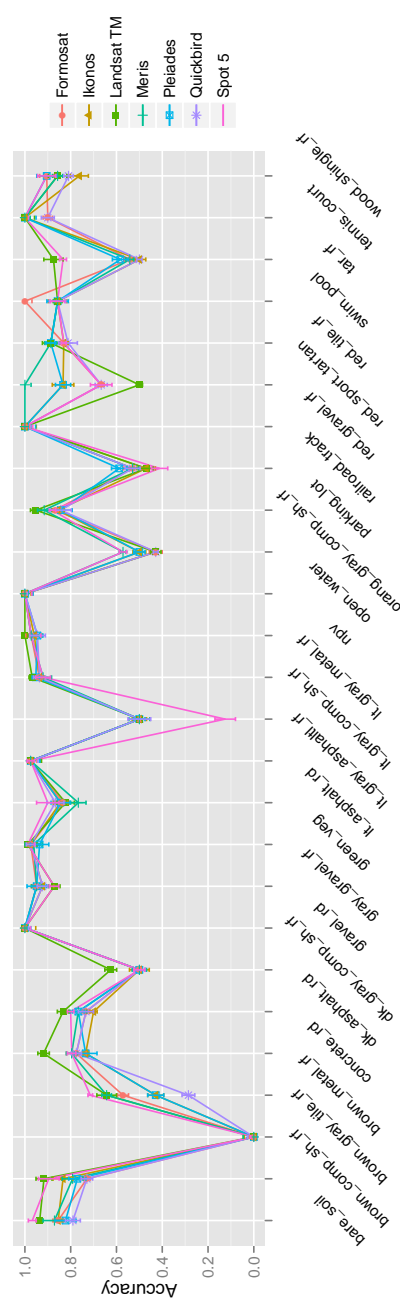

(b) Level 4

Figure 6. Classification accuracy per class for HEROLD Level 3 (a) and Level 4 (b)

\subsection{Spectral index assessment}

Spectral indexes are non linear combinations of bands which are used to highlight some specific features in images (vegetation, soil, buildings, water, etc.). Simulation can be used to evaluate if the addition of a spectral index improves the separability of the classes along with the classification accuracy. We give in this section a simple example with the well-known Normalized Difference Vegetation Index (NDVI). We classified the dataset generated for Ikonos, Pleiades, Quickbird and Spot 5-HRV with and without the addition of the NDVI.

The results presented in Table 8 reveal that the addition of the NDVI tends to increase the classification accuracy for most of the datasets but not for all (e.g. NCGIA). In this experiment, Spot 5-HRV does not seem to leverage the information provided by the NDVI, except for the Herold dataset where the NDVI seems informative for all the sensors. 
Table 8. Evaluation of the influence of the NDVI channel on the different sensors.

\begin{tabular}{lllllllllll}
\hline & Aster 1 & Aster 2 & Aster 3 & NCGIA & USGS & Herold1 & Herold 2 & Herold 3 & Herold 4 \\
\hline Ikonos & 83.333 & 53.333 & 45.185 & 59.398 & 77.093 & 96.444 & 91.527 & 85.669 & 85.356 & \\
+ NDVI & $83.704 \nearrow$ & $54.444 \nearrow$ & $45.556 \nearrow$ & $60.902 \nearrow$ & $76.860 \searrow$ & 97.490 & 92.364 & $88.912 \nearrow$ & $87.866 \nearrow$ \\
Pleiades & 92.222 & 61.111 & 50.000 & 62.406 & 77.661 & 96.548 & 91.841 & 86.611 & 86.715 \\
+ NDVI & $93.704 \nearrow$ & $61.852 \nearrow$ & $51.852 \nearrow$ & $57.895 \searrow$ & $79.064 \nearrow$ & $97.385 \nearrow$ & $91.213 \searrow$ & $88.389 \nearrow$ & $88.912 \nearrow$ \\
Quickbird & 84.444 & 51.481 & 44.815 & 61.654 & 75.930 & 96.444 & 90.586 & 85.146 & 85.251 \\
+ NDVI & $84.074 \searrow$ & $55.926 \nearrow$ & $45.185 \nearrow$ & $60.150 \searrow$ & $76.744 \nearrow$ & $97.803 \nearrow$ & $91.527 \nearrow$ & $87.971 \nearrow$ & $88.598 \nearrow$ \\
Spot 5-HRV & 89.259 & 57.778 & 50.741 & 63.158 & 77.544 & 96.757 & 91.632 & 88.180 & 88.389 \\
+ NDVI & $87.037 \searrow$ & $56.667 \searrow$ & $44.074 \searrow$ & $60.150 \searrow$ & $78.363 \nearrow$ & $96.967 \nearrow$ & $92.259 \nearrow$ & $88.285 \nearrow$ & $88.598 \nearrow$ \\
\hline
\end{tabular}

Table 9. Evaluation of the influence of the SWIR channel on the Spot 5-HRV and Pleiades sensors.

\begin{tabular}{|c|c|c|c|c|c|c|c|c|c|}
\hline & Aster 1 & Aster 2 & Aster 3 & NCGIA & USGS & Herold1 & Herold 2 & Herold 3 & Herold 4 \\
\hline Pleiades & 92.222 & 61.111 & 50.000 & 62.406 & 77.661 & 96.548 & 91.841 & 86.611 & 86.715 \\
\hline$+\mathrm{MIR}$ & 88.889 & $62.963 \nearrow$ & $49.630 \searrow$ & $62.406 \rightarrow$ & $78.246 \nearrow$ & $96.967 \nearrow$ & $92.992 \nearrow$ & $88.598 \nearrow$ & 89.121 \\
\hline Spot 5-HRV & 89.259 & 57.778 & 50.741 & 63.158 & 77.544 & 96.757 & 91.632 & 88.180 & 88.389 \\
\hline - MIR & 87.778 & 55.556 & 48.889 & $57.143 \searrow$ & 75.322 & 95.816 & 90.795 & $83.054 \searrow$ & 84.623 \\
\hline
\end{tabular}

\subsection{Spectral band removal/addition assessment}

Simulation can also be used to evaluate if the addition of a band to a sensor is relevant for a certain type of application. It allows to evaluate in advance the potentialities of the new multispectral sensor, and provide an opportunity to try variations in the original spectral response, and to adjust the RSR to achieve better results for the multispectral sensor objectives.

To illustrate this, we choose to evaluate how the Pleiades sensor reacted to the addition of the Short-Wave Infra Red band (SWIR) and how the Spot 5-HRV sensor reacted to its removal. The results are presented in Table 9 and Figure 7. The addition of the SWIR band to Pleiades sensor did not seem to improve the results in this specific experiment. However its removal to the Spot 5-HRV sensor greatly influenced the classification results as the accuracy are always lower without this band. These results can help to design or adjust the definition of spectral bands for future satellites program.

\section{Conclusion}

In this paper, we presented how sensor simulation could be used to evaluate the ability of a sensor to discriminate spectral variation of land covers and materials. This simulation consisted in converting precise spectra extracted from spectral libraries into spectra at a coarser resolution. Seven of the most common multispectral sensors (i.e. Ikonos, Pleiades, Quickbird, Formosat-2, Landat, Spot 5 and Meris) were simulated and compared using four spectral libraries totalizing around 2500 spectra. A comparison using the reduced spectra was performed to assess their ability to discriminate and classify different land covers and materials. The presented results provide important insights on sensor discrimination abilities according to their Relative Spectral Response (RSR). Furthermore, different ways to use the simulation to compare and understand differences between sensors were presented. For example, we showed that small differences in the definition of a band can lead to very different classification results (Sect 5.1) (e.g. Quickbird (84.4\%) and Pleiades $(92.2 \%)$ explained by a difference in the NIR band). We also highlighted that depending on the level of details (e.g. number of classes to differentiate), some sensors were more recommended than others (Sect 5.1) (e.g. Landsat performed well in urban areas thanks to its specific bands). We also showed that the potential 


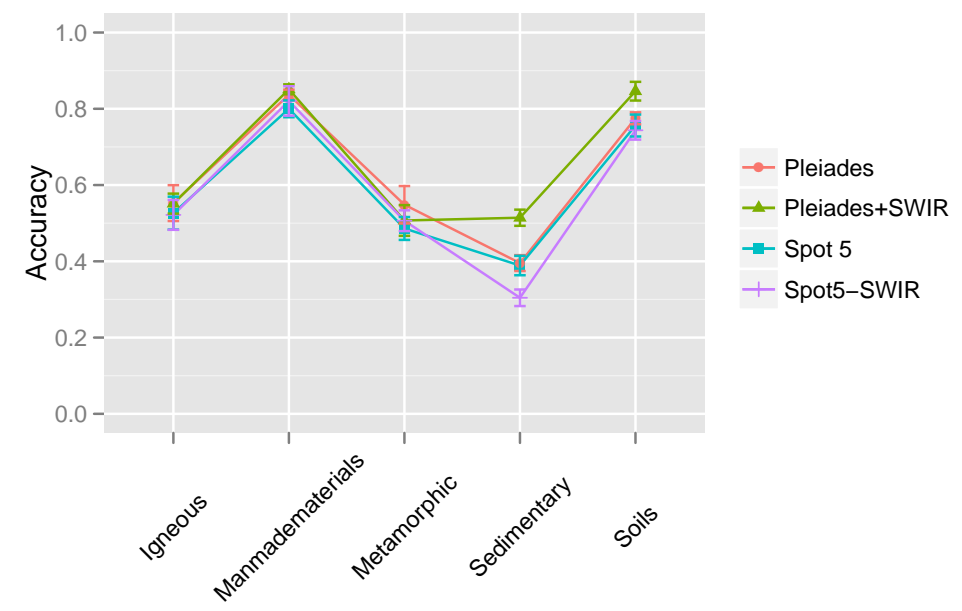

(a) Classification accuracy per class for ASTER/JHU Level 2

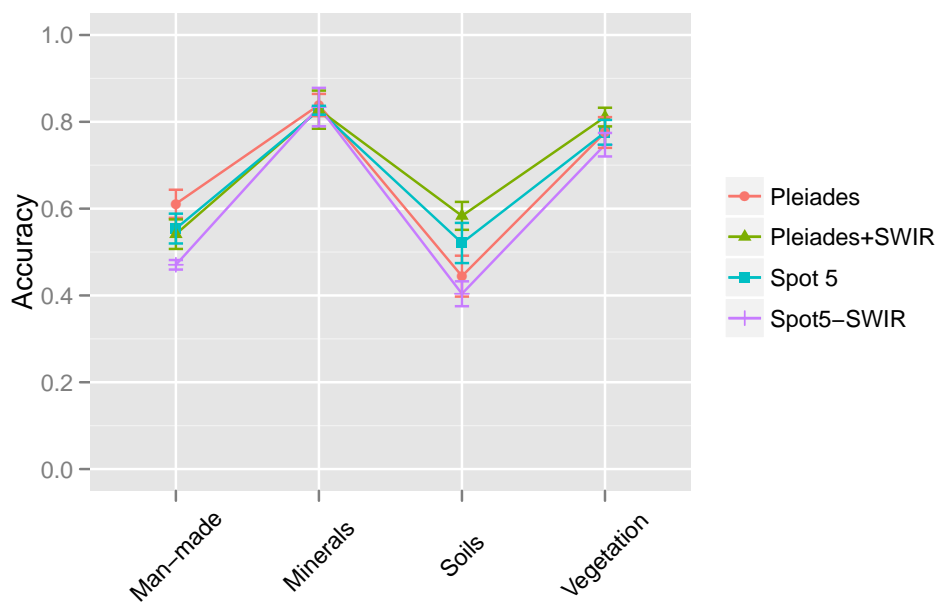

(b) Classification accuracy per class for USGS

Figure 7. Influence of adding/removing the SWIR channel to/from Pleiades and SPOT5 on the classification accuracy. (a) Classification accuracy per class for ASTER/JHU Level II and (b) classification accuracy per class for USGS.

improvement of classification accuracy using a spectral index (e.g. NDVI) could be evaluated through simulation (Sect 5.2). Along with helping to understand existing sensors, we highlighted that simulation could be used to help creating new ones (Section 5.3). Indeed, the tedious process of sensor design could be greatly backed by early simulation which could help to design specific RSR.

The results presented in this study have to be balanced by the relative low number of spectra present in the used spectral librairies and their relevance for different applications. Indeed, spectral libraries are expensive to develop and consequently, are rarely freely distributed Furthermore, it is worth noticing that the simulation presented in this study did not take into account several important factors like the impact of atmosphere, the spatial resolution of the sensor, the mixture of spectra, the acquisition noise, etc. Indeed, remote sensing images often exhibit mixed pixels which are composed of a mixture of several pure spectra and some noise. While 
this study does not address directly this issue, it provides a first comparison of sensor discrimination ability focusing only on spectral differences. Further work is necessary to improve the simulation task to take into account more parameters in order to be closer to the reality and the complexity of remote sensing data. However, this kind of simulation is still an unexplored wilderness and possesses a great potential for various remote sensing applications.

\section{References}

Baldridge, A. M., S. J. Hook, C. I. Grove, and G. Rivera. 2008. "The ASTER Spectral Library Version 2.0." Remote Sensing of Environment .

Bo, Yanchen, Jinfeng Wang, and Xiang Li. 2005. "Exploring the scale effect in land cover mapping from remotely sensed data: the statistical separability-based method." International Geoscience and Remote Sensing Symposium .

Bruzzone, L., F. Roli, and S. B. Serpico. 1995. "An extension of the Jeffreys-Matusita distance to multiclass cases for feature selection." IEEE Transactions on Geoscience and Remote Sensing 33: 1318-1321.

Bruzzone, L., and S. B. Serpico. 2000. "A technique for feature selection in multiclass problems." International Journal of Remote Sensing 21 (3): 549-563.

Chander, G., D.J. Meyer, and D.L. Helder. 2004. "Cross calibration of the Landsat-7 ETM+ and EO-1 ALI sensor." IEEE Transactions on Geoscience and Remote Sensing 42 (12): 2821-2831.

Clark, R.N., G.A. Swayze, R. Wise, E. Livo, T. Hoefen, R. Kokaly, and S.J. Sutley. 2007. "USGS digital spectral library splib06a." U.S. Geological Survey, Digital Data Series 231 .

Clark, Roger N., Gregg A. Swayze, K. Eric Livo, Raymond F. Kokaly, Trude V. V. King, J. Brad Dalton, J. Sam Vance, Barnaby W. Rockwell, Todd Hoefen, and Robert R. McDougal. 2002. "Synthesis of Multispectral Bands from Hyperspectral Data: Validation Based on Images Acquired by AVIRIS, Hyperion, ALI, and ETM+." AVIRIS Workshop

Davis, Shirley M., and Philip H. Swain. 1978. Remote Sensing: The Quantitative Approach. McGraw-Hill International Book Company.

De Backer, S., P. Kempeneers, W. Debruyn, and P. Scheunders. 2005. "A band selection technique for spectral classification." Geoscience and Remote Sensing Letters, IEEE 2 (3): 319-323.

Ferwerda, Jelle G, Simon D. Jones, and Marcus Reston. 2006. "A free online reference library for hyperspectral reflectance signatures." SPIE Newsroom .

Forestier, G., C. Wemmert, and P. Gancarski. 2008. "Multi-source Images Analysis Using Collaborative Clustering." EURASIP Journal on Advances in Signal Processing - Special issue on Machine Learning in Image Processing 11p.

Franke, J., V. Heinzel, and G. Menz. 2006. "Assessment of NDVI- Differences Caused by Sensor Specific Relative Spectral Response Functions." IEEE International Geoscience and Remote Sensing Symposium 1138-1141.

Govender, M., K. Chetty, and H. Bulcock. 2007. "A review of hyperspectral remote sensing and its application in vegetation and water resource studies." Water SA 33 (2): 145-152.

Green, Robert O., and Masanobu Shimada. 1997. "On-orbit calibration of a multi-spectral satellite sensor using a high altitude airborne imaging spectrometer." Advances in Space Research 19: 1387-1398.

Gunal, S., and R. Edizkan. 2008. "Subspace based feature selection for pattern recognition." Information Sciences 178 (19): 3716-3726.

Gunala, Serkan, and Rifat Edizkanb. 2008. "Subspace based feature selection for pattern recognition." Information Sciences 178: 3716-3726.

Guo, Baofeng, R. I. Damper, Steve R. Gunn, and J. D. B. Nelson. 2008. "A fast separability- 
based feature-selection method for high-dimensional remotely sensed image classification." Pattern Recognition 41 (5): 1653-1662.

Heidena, Uta, Karl Segl, Sigrid Roessner, and Hermann Kaufmann. 2007. "Determination of robust spectral features for identification of urban surface materials in hyperspectral remote sensing data." Remote Sensing of Environment 111: 537-552.

Herold, M., M.E. Gardner, and D.A. Roberts. 2003. "Spectral resolution requirements for mapping urban areas." IEEE Transactions on Geoscience and Remote Sensing 41 (9): 1907-1919.

Herold, Martin, Dar A. Roberts, Margaret E. Gardner, and Philip E. Dennison. 2004. "Spectrometry for urban area remote sensing : Development and analysis of a spectral library from 350 to $2400 \mathrm{~nm} . "$ Remote Sensing of Environment 91: 304-319.

Hueni, A., J. Nieke, J. Schopfer, M. Kneubhler, and K Itten. 2009. "The spectral database SPECCHIO for improved long-term usability and data sharing." Computers \& Geosciences 35: 557-565.

Jarecke, P., P. Barry, J. Pearlman, and B. Markham. 2001. "Aggregation of Hyperion hyperspectral spectral bands into Landsat-\&ETM+ spectral bands." IEEE International Geoscience and Remote Sensing Symposium 6: 2822-2824.

Kavzoglu, T. 2004. "Simulating Landsat ETM+ imagery using DAIS 7915 hyperspectral scanner data." International journal of remote sensing 25 (22): 5049-5067.

Kittler, Josef, Mohamad Hatef, Robert P. W. Duin, and Jiri Matas. 1998. "On Combining Classifiers." IEEE Transaction on Pattern Analysis and Machine Intelligence 20 (3): 226-239.

Kruse, Fred A. 2009. "Improving multispectral mapping by spectral modeling with hyperspectral signatures." Journal of Applied Remote Sensing 3.

Lai, Carmen, Marcel J. T. Reinders, and Lodewyk Wessels. 2006. "Random subspace method for multivariate feature selection." Pattern Recognition Letters 27 (10): 10671076 .

Lillesand, Thomas M., and Ralph W. Kiefer. 1994. Remote Sensing and Image Interpretation. John Wiley \& Sons.

Liua, Huawen, Jigui Sun, Lei Liua, and Huijie Zhang. 2009. "Feature selection with dynamic mutual information." Pattern Recognition 42: 1330-1339.

Maghsoudi, Y., M.J.V. Zoej, and M. Collins. 2011. "Using class-based feature selection for the classification of hyperspectral data." International Journal of Remote Sensing 32 (15): 4311-4326.

Masunaga, H., T. Matsui, W. Tao, A.Y. Hou, C.D. Kummerow, T. Nakajima, P. Bauer, W.S. Olson, M. Sekiguchi, and T.Y. Nakajima. 2010. "Satellite Data Simulator Unit (SDSU): A multi-sensor, multi-spectral satellite simulator package." Bulletin of the American Meteorological Society .

Meyer, D.J., and G. Chander. 2007. "The effect of variations in relative spectral response on the retrieval of land surface parameters from multiple sources of remotely sensed imagery." IEEE International Geoscience and Remote Sensing Symposium 5150-5153.

Mi-Hyun, Park, and Stenstrom Michael K. 2008. "Classifying environmentally significant urban land uses with satellite imagery." Journal of Environmental Management 86 (1): $181-192$.

Pandya, MR, RP Singh, KN Chaudhari, KR Murali, AS Kirankumar, VK Dadhwal, and JS Parihar. 2007. "Spectral characteristics of sensors onboard IRS-1D and P6 satellites: Estimation and their influence on surface reflectance and NDVI." Journal of the Indian Society of Remote Sensing 35 (4): 333-350.

Ranson, K. J., K. Kovacs, G. Sun, and V. I. Kharuk. 2003. "Disturbance recognition in the boreal forest using radar and Landsat-7." Canadian Journal of Remote Sensing 29 (2): 271-285.

Richards, John A, and Xiuping Jia. 2006. Remote Sensing Digital Image Analysis. springer ed.

Riedmann, M., and E.J. Milton. 2003. "Supervised band selection for optimal use of data from airborne hyperspectral sensors." IEEE Geoscience and Remote Sensing Symposium 3: $1770-1772$. 
Rivard, B., D. Rogge, F. Feng, B. Grant, and J. Pardie. 2011. "Hyperspectral sensing in support of mineral exploration in northern latitude remote regions." Geological Association of Canada Minerological Association of Canada .

Salvatore, Enio, Carmine Esposito, Thelma Krug, and Robert Green. 1999. "Simulation of the Spectral Bands of the CDD and WFI Cameras of the CBERS Satellite using AVIRIS Data." .

Segl, K., L. Guanter, H. Kaufmann, J. Schubert, S. Kaiser, B. Sang, and S. Hofer. 2010. "Simulation of spatial sensor characteristics in the context of the EnMAP hyperspectral mission." IEEE Transactions on Geoscience and Remote Sensing 48 (7): 3046-3054.

Sotoca, J.M., F. Pla, and J.S. Sanchez. 2007. "Band Selection in Multispectral Images by Minimization of Dependent Information." IEEE Transactions on Systems, Man, and Cybernetics, Part C: Applications and Reviews 37 (2): 258-267.

Teillet, P.M., K. Staenz, and D.J. William. 1997. "Effects of spectral, spatial, and radiometric characteristics on remote sensing vegetation indices of forested regions." Remote Sensing of Environment 61 (1): 139 - 149.

Trishchenko, Alexander P. 2009. "Effects of spectral response function on surface reflectance and NDVI measured with moderate resolution satellite sensors: Extension to AVHRR NOAA-17, 18 and METOP-A." Remote Sensing of Environment 113 (2): 335 $-341$.

Wemmert, C., A. Puissant, G. Forestier, and P. Gancarski. 2009. "Multiresolution Remote Sensing Image Clustering." IEEE Geoscience and Remote Sensing Letters 6 (3): 533 537. 